\title{
Functional and Esthetic Correction of Severe Localized Pregnancy-Induced Gingival Enlargement Associated with Capillary Hemangioma
}

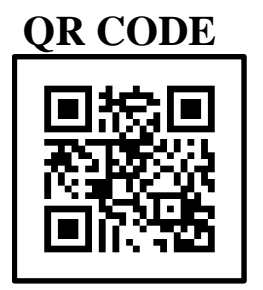

\section{PAWAN KUMAR', SAINDHYA TORA SONOWAL², JITU CHAWLA³}

Gingival enlargement is a clinical condition that has been directly associated with specific local or systemic conditions. Pregnancy has been considered an attributing factor which increases the susceptibility to gingival enlargement. It is also considered as a risk factor for periodontitis because of its ability to allow proliferation of specific microorganisms and affect host immunological response. This paper presents a rare case report of capillary hemangioma on attached gingiva of anterior maxilla in an adult female which initiated when she was in her 1oth week of gestation. After parturition, gingival enlargement further progressed and caused functional and aesthetic problem. Enlargement did not resolve even after non-surgical therapy; therefore, surgical excision of the entire enlargement was preformed. Histopathological examination revealed capillary hemangioma. No evidence of malignancy was seen. No recurrence was seen even after 2 years of follow-up.

\section{INTRODUCTION}

Gingival enlargement or gingival overgrowth is a clinical term, associated with local or systemic conditions and has been classified according to etiology as inflammatory, drug induced, associated with systemic diseases, and neoplastic. Enlargement in pregnancy is conditional, due to increase in levels of progesterone and estrogen, plaque remaining the main etiology.

This paper presents a case report of capillary hemangioma on attached gingiva which presented as gingival overgrowth of anterior maxilla and initiated when she was in her 1oth week of gestation. After parturition, gingival enlargement further progressed and caused functional as well as aesthetic problem. After non-surgical therapy, overgrowth did not resolve, so surgical management was performed. Histopathological examination revealed capillary hemangioma with no malignancy. No recurrence was seen after 2 years of follow-up.

Prevalent diseases of the oral cavity include gingivitis, periodontitis and dental caries. ${ }^{1}$ Onset and progression of periodontitis varies among individuals depending on both host and environmental factors.

Among others increase in sex hormone level is host risk factor inducing proliferation of specific periodontal microorganisms ${ }^{2}$ and affecting host immune response. ${ }^{3}$ Pregnancy gingivitis is a common finding which is prevalent in almost 30$100 \%$ of women. ${ }^{4}$ Frequent gingival changes are observed which leads to deterioration of gingival health at the time of conception.

Therefore a positive control of periodontal pathogen is crucial in the optimal management of such conditions. Gingival enlargement is a clinical condition and may present with regular or irregular surface features, fibrosis or edema, localized or generalized, and may be of mild or severe degree.

Enlargement often starts in the interdental papilla and may coalesce to involve the marginal and attached gingiva. The cause of gingival enlargement is dental plaque and modified by systemic conditions. ${ }^{5}$ In pregnancy there is an increase in gingivitis due to increase in hormonal levels beginning with increase in gonadotropin levels which is maintained throughout 4-8 months of pregnancy and also in the proportion of Prevotella intermedia. ${ }^{6,7}$ Increased levels of hormones during gestation also stimulates increased gingival vascular permeability and edema, respectively. ${ }^{8}$ Gingivitis decreases after parturition. This paper presents a case report of localized, severe, gingival enlargement associated 
with capillary hemangioma.

\section{CASE REPORT}

A 26-year-old woman reported with enlarged gums of the upper right jaw since 4 months.

Her medical history revealed that the patient was in her 26th week of gestation. There was no history of any systemic disease and/or condition. She had no known drug allergy and was not under any medication. Her vital signs were within normal limits. History of present illness revealed that she was pregnant at the time of onset of swelling and the swelling had grown to present size.

Her intraoral examination revealed pinkish red, fibrotic, bilobular, localized enlarged gingiva extending from mesial of 11 to distal half of 14 . In the anterioposterior aspect it was extending from vestibular depth to the anterior one third of hard palate. The oral hygiene of the patient was poor and the enlargement was a functional and aesthetic problem for the patient.

The patient's treatment consisted of patient education and oral hygiene instructions, mechanical debridement and chlorhexidine mouth rinses. Patient was kept under observation and was advised to review after parturition. The patient reported after 6 weeks of parturition. The gingival enlargement was noted to be further increased when compared to 26th week of gestation.

Before beginning of the treatment a written consent was obtained from the patient. Mechanical debridement was performed and the patient was given oral hygiene instructions and medications. After the non-surgical therapy, reevaluation of the patient was done. The gingival condition improved but improvement in gingival enlargement was not noted; therefore, surgical management of the growth was planned (Figures 1-3). Gingivectomy was performed under local anesthesia and the excised tissue was sent for histopathological examination. Scaling and root planing was done in the area and periodontal dressing was given.

The patient was given oral hygiene instructions, medications and recalled after 1 week. Histopathological examination showed fibrous connective tissue covered partly by inflammatory exudates and partly by squamous epithelium. Stroma showed vascular spaces with blood which were intercommunicating, thin walled and of variable size lined by endothelium suggesting capillary haemangioma. There were also chronic inflammatory cells infiltrating the area. Malignancy was absent. The case was followed regularly and no recurrence was observed till 2 years of follow-up(Figures 4-6).

\section{DISCUSSION}

Vascular anomalies comprise of a wide range of varied group of tumors and malformations. ${ }^{9}$ Hemangioma comprises of various group of clinical benign vascular lesions. ${ }^{10}$ They are characterized by rapid endothelial cell proliferation, followed by involution over time.

The proliferating masses of vessels do not undergo malignant transformation. Though they usually develop in children, occasionally older age individuals are affected. ${ }^{11}$ Clinically the incidence is higher in females (65\%) than males $(35 \%){ }^{12}$ Based on the size of vascular spaces and histology, hemangiomas are classified into capillary and cavernous types.

Cavernous hemangiomas consists of deep, irregular, dermal tangles of large thin walled vessels of sinusoids separated by scanty connective tissue surrounded by discontinuous layer of endothelial cell whereas capillary hemangiomas have numerous proliferating, small, thin walled blood filled vessels composed of single layer of flattened or plump endothelial cells, surrounded by discontinuous layer of pericytes and reticular fibers.

Capillary hemangioma $(\mathrm{CH})$ as a term has been commonly practiced to describe a large number of vasoactive tumours (VFT). $\mathrm{CH}$ exhibits both, a proliferative phase and an involution phase, where as vascular malformations are more stable and fail to regress. Clinically hemangiomas present as a soft tissue mass with variable size, either smooth/ lobulated, sessile/pedunculated.

The color varies from deep red to purple which blanches on pressure application. Most $\mathrm{CH}$ involutes with time, but a small percentage does not, and may present with complication requiring 
surgical management. $\mathrm{CH}$ is a common soft tissue tumor of head and neck, ${ }^{13}$ but is a rare and uncommon pathologic entity in the oral cavity. They are occasionally seen on oral mucosa.

Occurrence on gingiva is extremely rare. These growths often appear to arise from interdental papilla and spread laterally to involve adjacent tissue. $^{14} \mathrm{CH}$ clinically resembles pyogenic granuloma though they differ in histological findings. Even though it is asymptomatic, its site and size may require immediate careful intervention. It often presents as a diagnostic dilemma to the clinician.

This necessitates biopsy of such lesions for establishing a definite diagnosis and proper management and prevention of various complications. Most importantly, the surgical excision of $\mathrm{CH}$ should be performed with caution as there can be complication of intra- and post operative hemorrhage. There is also high risk of recurrence that necessitates longer follow up of the site.

Gingival enlargement associated with pregnancy usually resolves after parturition but if they still persist, such enlargements should be treated with paid special attention and care. After surgical excision the biopsy must be sent for Histopathological evaluation to rule out other differential diagnosis which may include benign or malignant neoplastic lesions.

It is also important to keep a regular follow up and judicious management to any vascular condition associated with pregnancy due to the added component of hormonal influence.

\section{REFERENCE}

1. Petersen PE. The World Oral Health Report 2003: continuous improvement of oral health in the 21st century-the approach of the WHO Global Oral Health Programme. Commun Dent Oral Epidemiol 2003;31(Suppl 1):3-23.

2. Raver-Durlacher JE, van Steenbergen TMJ, van der Velden U, de Graaff J, Abraham-Inpijn L. Experimental gingivitis during pregnancy and postpartum: clinical, endocrinological, and microbiological aspects. J Clin Periodontal 1994;21:549-58.

3. Ferris GM. Alteration in female sex hormones, their effects on oral tissues and dental treatment. Compendium 1993;14:1558-64, 1566-70.

4. Mariotti A. Sex steroid hormones and cell dynamics in the periodontium. Crit Rev Oral Biol Med 1994;5:27-53.

5. Newman MG, Takei HH, Carranza FA. Carranza's clinical periodontology. 9th edn. Philadelphia: WB Saunders, 2002:279-96.

6. Amar S, Chung KM. Influence of hormonal variation on the periodontium in women. Periodontol 200o. 1994;6:79-87.

7. Tilakaratne A, Soory M, Ranasinghe AW, et al. Periodontal disease status during pregnancy and 3 months post-partum, in a rural population of Sri Lankan women. J Clin Periodontol 2000;27:787-92.

8. Raber-Durlacher JE, Leene W, Palmer-Bouva $\mathrm{CC}$, et al. Experimental gingivitis during pregnancy and post-partum: immunohistochemical aspects. J Periodontol 1993;64:211-8.

9. Chang MW. Updated classification of hemangiomas and other vascular anomalies. Lymphat Res Biol 2003; 1: 259-65.

10. Enzinger FM, Weiss SW. Soft tissue tumors. 3rd ed. St. Louis: Mosby; 1995.p.581-6.

11. Silverman RA. Hemangiomas and vascular malformations. Pediatr Clin North Am 1991; 38:811-34.

12. Dilley DC, Siegel MA, Budnick S. Diagnosing and treating common oral pathologies. Pediatr Clin North Am 1991; 38:1227-64.

13. Warner M, Suen JY, Dinehart S. Treatment of hemangiomas of the head and neck. Laryngoscope 1992; 102:1123-32.

14. Carranza FA. Glickman's Clinical Periodontology. 1st ed. Philadelphia: WB Saunders Co.; 1990.p.35-51. 


\section{Source of support: Nil, Conflict of interest: None declared}

Cite this article as:

Kumar P, Sonowal ST, Chawla J. Functional and Esthetic Correction of Severe Localized Pregnancy-Induced Gingival Enlargement associated with Capillary

Hemangioma. Int Healthcare Res J 2017;1(8):248-251.

\section{AUTHOR AFFILIATIONS:}

1. MDS (Periodontics) Ph.D Scholar, Tezpur Central University, Assam

2. MDS (Periodontics), Registrar, Department of Dentistry, Tezpur Medical College, Tezpur, Assam

3. MDS (Oral and Maxillofacial Surgery), Department of Oral and Maxillofacial Surgery, Government Dental College, Nagpur, Maharastra

\section{Corresponding Author:}

Dr. Pawan Kumar

MDS Periodontics, Ph.D Scholar

Tezpur Central University, Assam

+91- 7528936969

drpkperio@gmail.com

\section{LEGENDS}

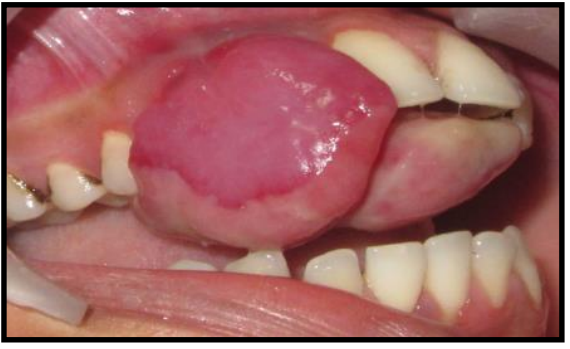

Figure 1. Lateral view of the enlargement (Pre-operative)

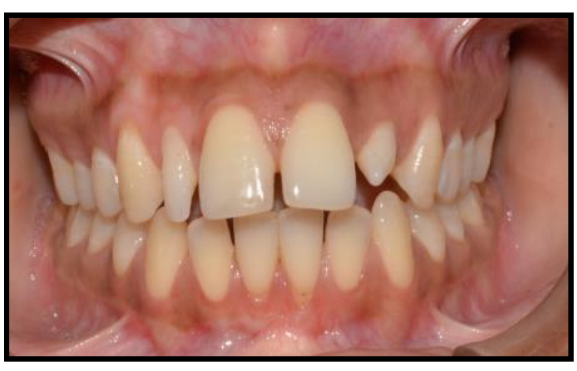

Figure 4. Anterior-posterior view (Post-operative)

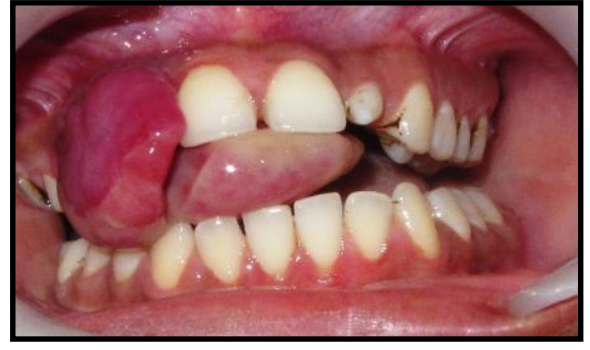

Figure 2. Anterior-posterior view of the enlargement (Pre-operative)

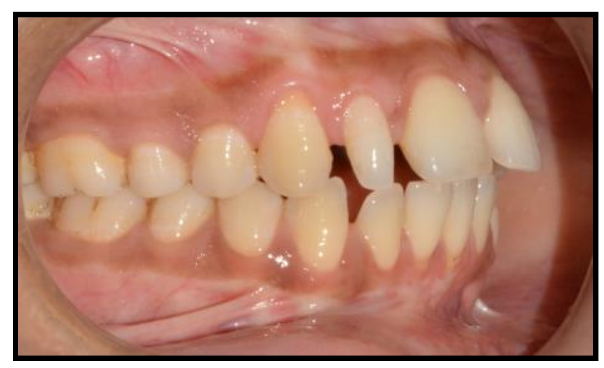

Figure 5. Lateral view (Post-operative)

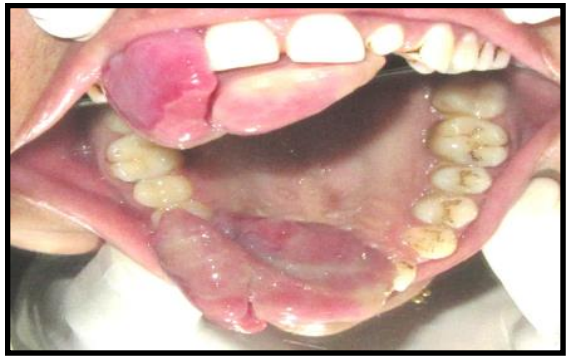

Figure 3. Extension of the lesion palatally (Pre-operative)

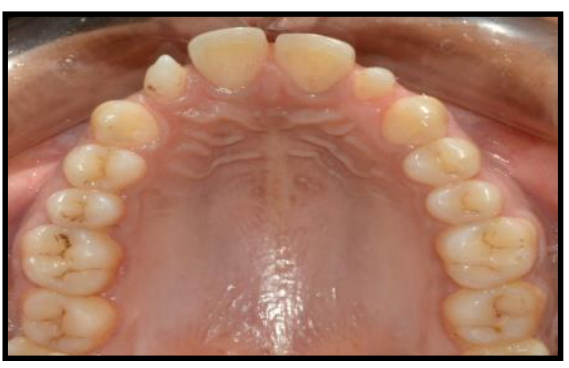

Figure 6. Palatal view (Post-operative) 